\title{
Novel Antiplatelet Agent Use for Acute Coronary Syndrome in the Emergency Department: A Review
}

\author{
M. Curial, E. Nath, and E. Lang \\ Department of Family Medicine, University of Calgary, 163214 Avenue NW, Calgary, AB, Canada T2N 1M7 \\ Correspondence should be addressed to M. Curial; mcurial@gmail.com
}

Received 11 October 2012; Revised 24 December 2012; Accepted 18 January 2013

Academic Editor: Ping-Yen Liu

Copyright (c) 2013 M. Curial et al. This is an open access article distributed under the Creative Commons Attribution License, which permits unrestricted use, distribution, and reproduction in any medium, provided the original work is properly cited.

\begin{abstract}
Background. Acute Coronary Syndrome (ACS) is a clinical condition encompassing ST Segment Elevation Myocardial Infarction (STEMI), Non-ST Segment Elevation Myocardial Infarction (NSTEMI), and Unstable Angina (UA) and is characterized by ruptured coronary plaque, ischemic stress, and/or myocardial injury. Emergency department (ED) physicians are on the front lines of ACS management. The role of new antiplatelet agents ticagrelor and prasugrel in acute ED management of ACS has not yet been defined. Objective. To critically review clinical trials using ticagrelor and prasugrel in the treatment of ACS and inform practitioners of their potential utility in treating ACS in the ED. Results. Trials on the efficacy of ticagrelor and prasugrel achieve statistical significance in decreasing composite endpoints in select patient populations. Conclusion. The use of ticagrelor and prasugrel as first line ED treatment of ACS is not well established. Current evidence supports the use of several agents with the final decision based on treatment protocols conjointly developed between cardiology and emergency medicine (EM). Further clinical trials involving head-to-head trials or comparisons of drug-based strategies are required to show superiority in reducing cardiac endpoints with regard to ED initiation of treatment.
\end{abstract}

\section{Introduction}

Acute Coronary Syndrome (ACS) is a clinical syndrome comprising ST Segment Elevation Myocardial Infarction (STEMI), Non-ST Segment Elevation Myocardial Infarction (NSTEMI), and Unstable Angina (UA). ACS is a common and important diagnosis that is often made in the emergency department by front-line physicians and rapid recognition and diagnosis of ACS, risk stratification, and appropriate treatment have been shown to decrease morbidity and mortality [1].

In the context of ACS treatment, dual antiplatelet therapy with ASA and clopidogrel (a P2Y12 receptor inhibitor) reduces rates of harmful cardiac events such as cardiovascular causes of death, myocardial infarction, and stroke [2]. However, with new agents undergoing evaluation in large clinical trials, acute care providers need to know if the new P2Y12 receptor inhibitor antiplatelet agents ticagrelor and prasugrel are clinically superior to the current standard clopidogrel.
It has been established that a defined percentage of the population exhibits high platelet activity despite the use of clopidogrel. This phenomenon occurs anywhere from 5\% to $44 \%$ of patients studied depending on the clopidogrel dose and patient population [3]. It is uncertain what level of platelet activity during ACS is related to harmful cardiovascular outcomes such as cardiovascular death, myocardial infarction, and stroke [4]. Ticagrelor and prasugrel have been demonstrated to reduce levels of platelet activation when compared to clopidogrel $[5,6]$ which could lead to reduced risk of thrombosis and improved artery or stent patency. However, there is debate as to which patients will gain the most clinical benefit from these costly and potentially harmful agents.

In this paper we will critically appraise the few large clinical trials that examine the use of ticagrelor or prasugrel in the treatment of ACS and highlight current treatment guidelines for ACS. Our focus will be on information relevant to EM and other acute care physicians. 


\section{The Novel Antiplatelet Agents}

Clopidogrel, prasugrel, and ticagrelor are all examples of P2Y12 receptor inhibitor antiplatelet medications that can be used in the treatment of ACS. Clopidogrel and prasugrel both are irreversible inhibitors of the P2Y12 receptors on the platelet surface. Platelets inhibited by these two agents are affected for the remainder of their lifespan, and therefore platelet aggregation returns to baseline within 5-10 days of discontinuation of either drug. Clopidogrel is a prodrug that is activated in the liver by cytochrome P450 enzymes and genetic variability in enzyme function is known to cause the medication to be less effective in individuals who cannot convert the drug to its active form. Prasugrel is a prodrug as well but appears to be effective in most individuals. In contrast ticagrelor is a reversible noncompetitive antagonist of the P2Y12 receptor; its action and the recovery of platelet function likely depend on the serum concentration of the drug. Ticagrelor is not a prodrug.

Of the three agents, clopidogrel has the longest onset of action at 2 hours after administration of the initial loading dose. Both ticagrelor and prasugrel cause inhibition of platelet activity (IPA) within 30 minutes of the initial loading dose, and their time to achieve maximal IPA is 4-8 hours [7]. Clopidogrel has the shortest half-life elimination of its active metabolite at 30 minutes. The half-life of ticagrelor and prasugrel is 9 and 7 hours on average, respectively.

Both ticagrelor and prasugrel show increased risk of bleeding. Additionally ticagrelor can cause dyspnea as an adverse effect in $10-14 \%$ of patients. Dyspnea usually occurs early in the course of treatment and is self-limited. Ticagrelor is contraindicated for use in patients who are taking medications that are strong CYP3A4 inhibitors; it is also contraindicated in those patients who have a history of intracranial hemorrhage. Prasugrel is contraindicated in patients with a history of transient ischemic attack (TIA) or stroke. Table 1 compares and contrasts these agents.

\section{Prasugrel}

Data on the efficacy of prasugrel in the treatment of ACS comes from two large, industry-sponsored clinical trials: TRITON-TIMI 38 [11] and TRILOGY ACS [12].

3.1. TRITON-TIMI 38. The major study comparing prasugrel to clopidogrel is TRITON-TIMI 38. It compared prasugrel (60 mg loading dose, $10 \mathrm{mg}$ daily maintenance dose) to clopidogrel (300 mg loading dose, $75 \mathrm{mg}$ daily maintenance dose) in a double-blind, double-dummy, randomized control clinical trial. The study population was moderate to high risk ACS patients scheduled for PCI; 13,604 patients were enrolled with 10074 representing the UA/NSTEMI range of the ACS spectrum and 3534 with STEMI. Eligibilitydefining risk was calculated using the TIMI risk score [13]. NSTEMI/UA patients could be enrolled within 72 hours of symptom onset and STEMI patients could be enrolled within 12 hours after onset of symptoms for patients undergoing primary PCI and within 14 days after onset of symptoms for those managed medically. 99\% of patients received PCI.
Patients were followed for a minimum of 6 months up to a maximum of 15 months.

The primary endpoint of this study was a composite of (1) death from cardiovascular cause, (2) nonfatal myocardial infarction, and (3) nonfatal stroke measured for an average of 14.5 months after randomization. Results were analyzed in an intention to treat analysis and showed the following:

(i) decreased rates of primary endpoint in the NSTEMI/UA group treated with prasugrel (hazard ratio, $0.82 ; 95 \%$ confidence interval (CI) 0.73 to $0.93 ; P=0.002$; could not calculate the number needed to treat (NNT) due to absent raw data),

(ii) decreased rates of primary endpoint in STEMI group treated with prasugrel (hazard ratio, $0.79 ; 95 \%$ CI 0.65 to $0.97 ; P=0.02$; could not calculate the number NNT due to absent raw data),

(iii) decreased rates of primary endpoints overall for all patients treated with prasugrel (hazard ratio, $0.81 ; 95 \%$ confidence interval, 0.73 to $0.90 ; P<0.001$; NNT 46). This can be further broken down into

(a) a nonsignificant decrease in death from cardiovascular causes for all patients treated with prasugrel (hazard ratio, 0.89; 95\% confidence interval $0.70-1.12 ; P=0.31$ ),

(b) a significant decrease in nonfatal MI for all patients treated with prasugrel, the primary driver of the composite endpoint (hazard ratio, 0.76 ; $95 \%$ confidence interval 0.67 to $0.85 ; P<$ 0.001; NNT 46),

(c) a non-significant increase in nonfatal stroke for all patients treated with prasugrel (hazard ratio, $1.02 ; 95 \%$ confidence interval, $0.71-1.45 ; P=$ $0.93)$.

The primary safety endpoint of this study was major bleeding as defined by TIMI major bleeding criteria. This showed

(i) a significant increase in the rate of non-CABGrelated major bleeding (hazard ratio, 1.32; 95\% CI $1.03-1.68 ; P=0.03$; number needed to harm $(\mathrm{NNH})$ 167) further broken down to

(a) a significant increase in the rate of lifethreatening bleeding (hazard ratio, 1.52; 95\% CI $1.08-2.13 ; P=0.01 ; \mathrm{NNH} 200)$,

(b) a significant increase in the rate of fatal bleeding (hazard ratio, $4.19 ; 95 \%$ CI 1.58-11.11; $P=$ 0.002 ; NNH 334),

(ii) a significant increase in the rate of bleeding requiring transfusion (hazard ratio, 1.34; 95\% CI 1.11-1.63; $P<$ $0.001 ; \mathrm{NNH} 100)$,

(iii) a significant increase in the rate of CABG-related major bleeding (hazard ratio, 4.73; 95\% CI 1.90$11.82 ; P<0.001 ; \mathrm{NNH} 10)$. 
TABLE 1: Pharmacologic properties of P2Y12 receptor inhibitors used in ACS.

\begin{tabular}{|c|c|c|c|}
\hline & Clopidogrel [8] & Ticagrelor [9] & Prasugrel [10] \\
\hline Class & Thienopyridine & Nucleoside analogue & Thienopyridine \\
\hline Prodrug & Yes & No & Yes \\
\hline Route & Oral & Oral & Oral \\
\hline Metabolism & Hepatic & Hepatic (CYP34A) & Intestinal, serum, hepatic \\
\hline Mechanism of action & $\begin{array}{l}\text { Active metabolite } \\
\text { IRREVERSIBLY inhibits P2Y12 } \\
\text { subtype of ADP receptors on the } \\
\text { platelet surface, which prevents } \\
\text { activation of GIIb/IIIa receptor } \\
\text { complex, thereby reducing } \\
\text { platelet activation and } \\
\text { aggregation; platelets blocked by } \\
\text { clopidogrel are affected for the } \\
\text { remainder of their lifespan } \\
(\sim 7-10 \text { days); note that genetic } \\
\text { variability of CYP2C19 may } \\
\text { preclude patients from the full } \\
\text { effect of drug }\end{array}$ & $\begin{array}{l}\text { REVERSIBLY and } \\
\text { noncompetitively binds the } \\
\text { P2Y12 subtype of ADP receptors } \\
\text { on the platelet surface, which } \\
\text { prevents ADP-mediated } \\
\text { activation of the GIIb/IIIa } \\
\text { receptor complex, thereby } \\
\text { reducing platelet aggregation; } \\
\text { due to reversible antagonism of } \\
\text { the P2Y12 receptor, recovery of } \\
\text { platelet function is likely to } \\
\text { depend on serum concentrations } \\
\text { of drug and its active metabolite }\end{array}$ & $\begin{array}{l}\text { Active metabolite } \\
\text { IRREVERSIBLY blocks the } \\
\text { P2Y12 subtype of ADP receptors } \\
\text { on the platelet, which prevents } \\
\text { activation of the GIIb/IIIa } \\
\text { receptor complex, thereby } \\
\text { reducing platelet activation and } \\
\text { aggregation; platelet aggregation } \\
\text { returns to baseline within 5-9 } \\
\text { days of discontinuation }\end{array}$ \\
\hline Onset of action (IPA) & $\begin{array}{l}300-600 \text { mg loading dose } \\
\text { detected within } 2 \text { hours }\end{array}$ & $\begin{array}{l}180 \text { mg loading dose } \sim 41 \% \text { within } \\
30 \text { minutes }\end{array}$ & $\begin{array}{l}60 \text { mg loading dose within } 30 \\
\text { minutes }\end{array}$ \\
\hline Time to maximal IPA & 6 hours after loading dose & $4-8$ hours after loading dose & 4-8 hours after loading dose \\
\hline $\begin{array}{l}\text { Half-life elimination of } \\
\text { active metabolite }\end{array}$ & $\sim 30$ minutes & $\sim 9$ hours & $\sim 7$ hours (range $2-15$ hours) \\
\hline Excretion & Renal (50\%), biliary (46\%) & Biliary & Renal $(\sim 68 \%)$, biliary $(\sim 27 \%)$ \\
\hline $\begin{array}{l}\text { Significant adverse } \\
\text { effects }\end{array}$ & None & Increased minor/major bleeding & Increased minor/major bleeding \\
\hline Contraindications & $\begin{array}{l}\text { Hypersensitivity, active bleeding, } \\
\text { significant liver impairment, and } \\
\text { cholestatic jaundice }\end{array}$ & $\begin{array}{l}\text { Hypersensitivity, active bleeding, } \\
\text { history of intracranial } \\
\text { hemorrhage, hepatic } \\
\text { impairment, concomitant use of } \\
\text { strong CYP3A4 inhibitors (e.g., } \\
\text { ketoconazole, clarithromycin, } \\
\text { ritonavir, atazanavir, nefazodone) }\end{array}$ & $\begin{array}{l}\text { Hypersensitivity, active bleeding, } \\
\text { history of TIA, or stroke }\end{array}$ \\
\hline
\end{tabular}

IPA: inhibition of platelet aggregation.

Because of the increased risk in bleeding, a post hoc analysis was conducted and found three specific subgroups in which the benefit from prasugrel did not outweigh harm:

(i) patients with a history of previous stroke or TIA showed statistically significant net harm (hazard ratio, 1.54 ; $95 \%$ CI $1.02-2.32 ; P=0.04)$,

(ii) patients 75 years old and older showed no benefit to treatment with prasugrel (hazard ratio, $0.99 ; 95 \% \mathrm{CI}$ $0.81-1.21 ; P=0.92$ ),

(iii) patients under 60 kilograms showed no benefit to treatment with prasugrel (hazard ratio, $1.03 ; 95 \% \mathrm{CI}$ $0.69-1.53 ; P=0.89$ ).

Data from this trial suggests clinical superiority of prasugrel over clopidogrel in preventing the composite cardiac endpoint when used in moderate to high risk patients with planned PCI. This superiority is mainly seen in preventing nonfatal myocardial infarction with little or no impact on rates of cardiac death and nonfatal stroke. For the purpose of this study, nonfatal MI was defined as "distinct from the index event and... defined by symptoms suggestive of ischemia/infarction, electrocardiographic data, cardiac biomarker, or pathologic evidence of infarction dependent on the clinical situation" [14].

The study also suggests that treatment with prasugrel results in a small but statistically significant increase in bleeding, especially fatal bleeding. These rates appeared higher in three subgroups: patients with previous stroke or TIA, patients 75 years old or older, and patients weighing less than $60 \mathrm{~kg}$. This information should serve as a caution when selecting patients likely to benefit from prasugrel administration and suggests avoiding this medication in the previously mentioned populations.

Critical appraisal of this study suggests several limitations in determining which antiplatelet agent should be used for the acute ACS patient presenting to the ED. First, the appropriate loading dose of clopidogrel is currently being questioned in the literature with many specialists advocating a larger $600 \mathrm{mg}$ loading dose as opposed to the $300 \mathrm{mg}$ dose used in 
this study [15-18]. Use of a potentially suboptimal comparator might have biased the outcomes reported.

It is worth noting that patients were administered the study medication at any point between randomization up to 1 hour after leaving the catheterization laboratory. It is not clear how results would change if patients were started on dual antiplatelet therapy at the time of diagnosis (pretreatment). ACCOAST [19] is a current clinical trial investigating the risks and benefits of pretreating patients with $30 \mathrm{mg}$ of prasugrel at the time of ACS diagnosis and $30 \mathrm{mg}$ more at the time of PCI versus $60 \mathrm{mg}$ at the time of PCI only. Results from this trial are expected in early 2013 and will be very relevant to ED physicians.

TRITON-TIMI 38 is only applicable to moderate and high risk patients scheduled for PCI. It is difficult to determine what benefit patients not undergoing PCI would experience in terms of efficacy and bleeding risk. TRILOGY ACS, described below, fills that gap in knowledge.

3.2. TRILOGY ACS. TRILOGY ACS is a recent study which examined the effect of prasugrel usage in UA and NSTEMI patients not undergoing revascularization. Patients were randomized in the study only after a decision for medical management without revascularization was made. In addition, patients must have been classified as high risk by possessing at least one of the following characteristics:

(1) age of at least 60 years old,

(2) presence of diabetes mellitus,

(3) previous myocardial infarction,

(4) previous revascularization with either PCI or coronary artery bypass grafting (CABG).

Patients were excluded if they had a history of TIA or stroke, PCI or CABG within 30 days, renal failure on dialysis, or concomitant anticoagulant treatment.

This study was designed to assess the efficacy of prasugrel (10 mg daily dose) versus clopidogrel (75 mg daily dose) in long-term maintenance therapy for ACS patients that did not receive revascularization and used the same composite endpoint as TRITON-TIMI 38. Patients were enrolled up to 10 days after treatment decision reducing the applicability of this paper to ED care.

Results of the primary analysis [12] of patients under 75 years old study show no statistically significant change in the rate of the primary endpoint between clopidogrel and prasugrel groups.

\section{Ticagrelor}

PLATO is the major study pitting ticagrelor against clopidogrel in ACS patients [20]. This industry-funded multicenter, randomized, double-blind, double-dummy trial compared ticagrelor (180 mg loading dose, $90 \mathrm{mg}$ bid maintenance dose) against clopidogrel (300-600 mg loading dose, $75 \mathrm{mg}$ daily thereafter). The study population included patients admitted to hospital with ACS, with or without ST segment elevation, with an onset of symptoms during the previous 24 hours. 18,624 patients were enrolled in this study with 9333 randomly assigned to ticagrelor and 9291 randomly assigned to the clopidogrel group.

The primary endpoint of this study was a composite of (1) death from vascular causes, (2) nonfatal myocardial infarction, and (3) nonfatal stroke. For the purpose of this study, non fatal MI was defined as follows:

(i) recurrent $\mathrm{MI}$ within 18 hours of a previous $\mathrm{MI}-$ defined as recurrent cardiac ischemic symptoms and a new ST elevation;

(ii) recurrent MI after 18 hours but before cardiac markers have returned to normal-defined as symptoms and reelevation of troponin or CK-MB of at least $50 \%$ over a previous value that was decreasing;

(iii) MI after cardiac biomarkers have returned to normal-defined as elevation of biochemical markers above the upper limit of normal with either ischemic symptoms at rest, ECG changes, or pathological findings of an acute $\mathrm{MI}$;

(iv) MI within 24 hours after PCI-defined as cardiac biomarkers $\geq 3 \mathrm{x}$ the local laboratory upper limit of normal from a normal or decreasing level and after CABG $\geq 10 \mathrm{x}$ the upper limit of normal or $\geq 5 \mathrm{x}$ with new $\mathrm{Q}$ waves.

ST segment elevation was described as the persistent elevation of the ST segment of $1 \mathrm{~mm}$ or more in two or more contiguous leads or a new left bundle branch block and the need for PCI.

Analyses were by intention to treat and results showed

(i) a significant decrease in the primary composite endpoint for patients receiving ticagrelor (hazard ratio 0.84 ; 95\% confidence interval $0.77-0.92 ; P<0.001$; NNT $=53$ ). This composite can be further broken down into

(a) a significant decrease in death from vascular causes for all patients treated with ticagrelor (hazard ratio, 0.79; 95\% CI 0.69-0.91; $P=$ 0.001; NNT 91),

(b) a significant decrease in nonfatal MI for all patients treated with ticagrelor (hazard ratio, 0.84; 95\% CI 0.75-0.95; $P=0.005$; NNT 91),

(c) a nonsignificant increase in nonfatal stroke for all patients treated with ticagrelor (hazard ratio, 1.17; 95\% CI 0.91-1.52; $P=0.22$ ).

The primary safety endpoint of this study was major bleeding as defined by the TIMI major bleeding criteria and/or a study-specific set of criteria. Overall there were no statistical differences between the two groups with regard to major or life-threatening bleeding. The only statistically significant differences in primary safety endpoints were

(i) a significantly higher instance of fatal intracranial bleeding in the ticagrelor group (11/9235 (0.1\%) versus 1/9186 (0.01\%), $P=0.02$; NNH 112), 
(ii) a significantly lower rate of nonintracranial fatal bleeds in the ticagrelor group $(9 / 9235(0.1 \%)$ versus 21/9186 (0.3\%), $P=0.03$; NNT 500).

When closer analyzed, a statistically significant difference in non-CABG-related major bleeding was seen as follows:

(i) as defined by study criteria (hazard ratio, $1.19 ; 95 \% \mathrm{CI}$ $1.02-1.38 ; P=0.03$; NNH 143) and

(ii) as defined by TIMI criteria (hazard ratio, 1.25 ; $95 \%$ CI $1.03-1.53 ; P=0.03$; NNH 167).

Additionally, premature discontinuation of the study drug due to adverse events occurred more frequently with ticagrelor than with clopidogrel (in $7.4 \%$ of patients versus $6.0 \% ; P<0.001)$.

Data from this trial suggests clinical superiority of ticagrelor over clopidogrel in preventing the composite primary endpoint; however, patients on ticagrelor were observed to have higher rates of major bleeding not related to CABG and more instances of fatal intracranial bleeding. Patients in the study also more frequently discontinued ticagrelor due to adverse effects.

A benefit of this study with regards to its relevance to ED physicians is that all patients were randomized within 24 hours of symptom onset. Additionally, both invasively managed and noninvasively managed patients were enrolled. This early randomization and administration of study drug as well as a broader study population is more applicable to EM management of ACS.

\section{Prasugrel versus Ticagrelor}

Absent from this body of research is a direct comparison of prasugrel to ticagrelor. Since the two medications have never been studied in a head-to-head trial, they are difficult to compare. Recently, an international group attempted to compare the efficacy of prasugrel to that of ticagrelor in an indirect meta-analysis with data on prasugrel coming from the TRITON-TIMI 38 trial and data for ticagrelor from the DISPERSE-2 and PLATO trials (Table 2) [21]. Final conclusions from this study suggest that both prasugrel and ticagrelor are superior to clopidogrel and that these two drugs have similar efficacy and safety profiles.

\section{Overview of Current Treatment Guidelines for ACS}

We feel that treatment of ACS should be based on easyto-apply protocols to help increase the speed of delivery of treatment and minimize medical errors in complex treatment regimens. Rigorously developed clinical practice guidelines are available from the European Cardiology Society and the American Society of Cardiology. We will focus our analysis on the suggested roles of prasugrel and ticagrelor.

6.1. American College of Cardiology/American Heart Association (ACC/AHA) Guidelines. The ACC/AHA created a widely used version of North American based ACS guidelines.
Guidelines for STEMI (written in 2004 [22], updated in 2007 [23] and again in 2009 [24]) and for NSTEMI/UA (2007 [25], updated 2011 [26] and 2012 [27]) are in existence.

The initial 2004 guidelines for STEMI recommended initial treatment with ASA as an antiplatelet agent. Additional antiplatelet agents were not recommended in the emergency department. Once diagnostic angiography had been performed, clopidogrel was recommended to be started for patients scheduled to undergo PCI.

In subsequent updates to this document, the role of antiplatelet agents was modified; one primary change focused on the use of thienopyridine antiplatelet agents. The 2009 update recommended the use of clopidogrel as soon as possible in patients that may receive primary or nonprimary PCI (class one recommendation, level of evidence C) or prasugrel as soon as possible for patients that will be receiving primary PCI (class one recommendation, level of evidence B). The guidelines do not address the role of ticagrelor.

The current ACC/AHA NSTEMI guidelines were very recently published in August of 2012 and prasugrel and ticagrelor play a large role in the management of ACS. Dual antiplatelet therapy is now recommended (class one recommendation, level of evidence varies based on presentation and treatment) with ASA and one of clopidogrel, prasugrel, or ticagrelor in both short- and long-term management of ACS. It is important to note that the guideline writing group did not recommend one P2Y12 receptor inhibitor over another.

6.2. European Cardiology Society (ESC) Guidelines. The European Cardiology Society produces clinical practice guidelines to help guide medical practitioners in the treatment of ACS. Similar to the ACC/AHA, they have separate documents for NSTEMI/ACS (2011 [28]) and for STEMI/Acute Myocardial Infarction (2012 [29]).

ECS STEMI guidelines recommend dual antiplatelet therapy with ASA (class 1 recommendation, grade B evidence) and an ADP receptor antagonist (class 1 grade A) as early as possible for patients with planned PCI. The key difference in the ECS guidelines when compared to the ACC/AHA guidelines is that prasugrel and ticagrelor are suggested as the preferred ADP receptor antagonists (class 1 grade $\mathrm{B}$ ) and suggest clopidogrel only when prasugrel and ticagrelor are contraindicated or unavailable.

The ECS also suggests dual antiplatelet therapy to be started as soon as possible in NSTEMI patients (class 1 grade A). Similar to their STEMI recommendations, the ECS suggests prasugrel or ticagrelor (both class 1 grade $B$ ) as the $\mathrm{ADP}$ receptor antagonists of choice and again suggests clopidogrel only when prasugrel and ticagrelor are contraindicated or unavailable (class one grade A). Additionally, the ECS guidelines suggest a $600 \mathrm{mg}$ loading dose of clopidogrel for patients scheduled for invasive management when this medication is chosen as the ADP receptor antagonist (class 1 grade $B$ ).

\section{Conclusion}

While it has been shown that both prasugrel and ticagrelor can decrease rates of composite cardiac endpoints in carefully 


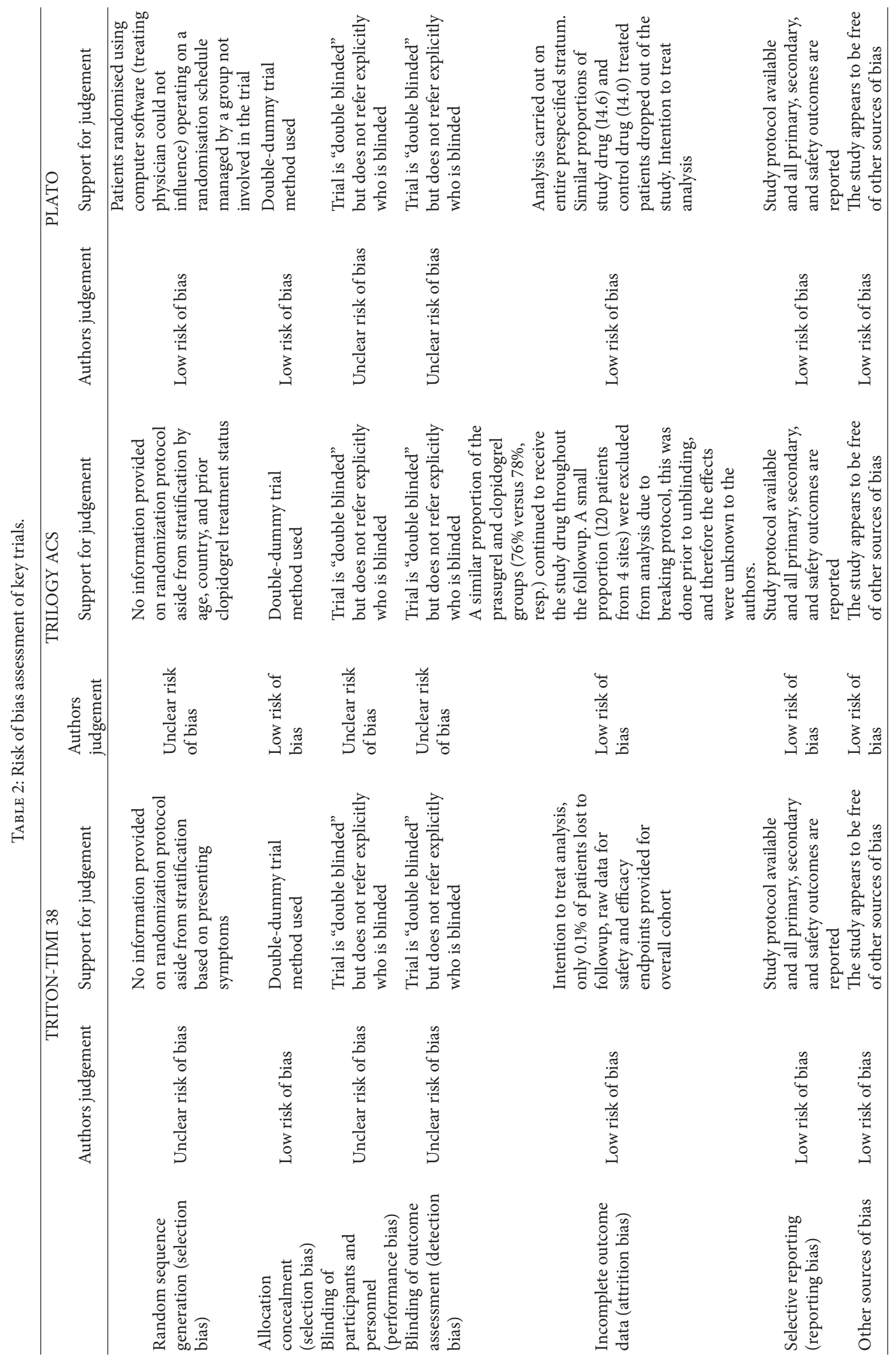


selected patients with ACS, the value of initiating treatment with these agents in the ED has not been clarified. Since much of the benefit of these drugs has been shown when given near the time of PCI and in long-term followup after ED discharge, we suggest that whenever possible decisions about antiplatelet agent choice be made in conjunction with the cardiology services providing immediate and long-term care. In general, patients that are at a low risk for bleeding will likely receive the greatest benefit from the new agents. High risk patients with planned PCI will likely benefit from prasugrel. The optimal timing of medication administration has not yet been established.

Having three appropriate agents for use in the current ACS guidelines causes a potential source of human error. With increased choice of therapeutic agents, treatment protocols become less standardized. This opens the door to potential omissions or delayed management as treatment decisions are being made. It is important to remember that current guidelines still focus on early recognition and treatment of ACS including appropriate revascularization and that providing new medications is not the primary goal of ED physicians.

Current evidence suggests improved efficacy of both prasugrel and ticagrelor over clopidogrel with quicker onset of action. However, these medications also have higher bleeding risks. Initiating treatment with these medications on all patients presenting to the ED with ACS has not been proven to decrease morbidity or mortality and we feel no compelling reason to recommend one agent over another for treatment of ACS in the ED. Instead, we feel the current evidence supports the use of any of these agents with the final decision based on patient presentation, national treatment guidelines, and local institutional treatment protocols. Optimal antiplatelet therapy is a rapidly evolving topic and the ED approach to antiplatelet therapy is likely to evolve in the upcoming months. However, we would like to stress that, despite medication advances, rapid recognition of ACS, risk stratification, and resuscitation when required are still the key roles of the EM physician in managing ACS.

\section{References}

[1] R. R. Arora and F. Rai, "Antiplatelet intervention in acute coronary syndrome," American Journal of Therapeutics, vol. 16, no. 5, pp. e29-e40, 2009.

[2] K. A. A. Fox, S. R. Mehta, R. Peters et al., "Benefits and risks of the combination of clopidogrel and aspirin in patients undergoing surgical revascularization for non- ST-elevation acute coronary syndrome: the clopidogrel in unstable angina to prevent recurrent ischemic events (CURE) trial," Circulation, vol. 110, no. 10, pp. 1202-1208, 2004.

[3] P. A. Gurbel and U. S. Tantry, "Clopidogrel resistance?" Thrombosis Research, vol. 120, no. 3, pp. 311-321, 2007.

[4] P. M. Vila, M. U. Zafar, and J. J. Badimon, "Platelet reactivity and nonresponse to dual antiplatelet therapy: a review," Platelets, vol. 20, no. 8, pp. 531-538, 2009.

[5] S. D. Wiviott, D. Trenk, A. L. Frelinger et al., "Prasugrel compared with high loading- and maintenance-dose clopidogrel in patients with planned percutaneous coronary intervention: the prasugrel in comparison to clopidogrel for inhibition of platelet activation and aggregation-thrombolysis in myocardial infarction 44 trial," Circulation, vol. 116, no. 25, pp. 2923-2932, 2007.

[6] P. A. Gurbel, K. P. Bliden, K. Butler et al., "Response to ticagrelor in clopidogrel nonresponders and responders and effect of switching therapies: The RESPOND Study," Circulation, vol. 121, no. 10, pp. 1188-1199, 2010.

[7] M. Valgimigli, M. Tebaldi, G. Campo et al., "Prasugrel versus tirofiban bolus with or without short post-bolus infusion with or without concomitant prasugreladministration in patients with myocardial infarction undergoing coronary stenting: the FABOLUS PRO, (Facilitation through Aggrastat by dropping or shortening Infusion Line in patients with ST-segment elevation myocardial infarction compared to or on top of PRasugrel given at loading dose) trial," Journal of the American College of Cardiology, vol. 5, no. 3, pp. 268-277, 2012.

[8] "Clopidogrel," in UpToDate, D. S. Basow, Ed., UpToDate, Waltham, Mass, USA, 2012.

[9] D. W. Marion, “Ticagrelor," in UpToDate, D. S. Basow, Ed., UpToDate, Waltham, Mass, USA, 2012.

[10] D. W. Marion, "Prasugrel," in UpToDate, D. S. Basow, Ed., UpToDate, Waltham, Mass, USA, 2012.

[11] S. D. Wiviott, E. Braunwald, C. H. McCabe et al., "Prasugrel versus clopidogrel in patients with acute coronary syndromes," The New England Journal of Medicine, vol. 357, no. 20, pp. 20012015, 2007.

[12] M. T. Roe, P. W. Armstrong, K. A. Fox et al., "Prasugrel versus clopidogrel for acute coronary syndromes without revascularization," The New England Journal of Medicine, vol. 367, no. 14, pp. 1297-1309, 2012.

[13] E. M. Antman, M. Cohen, P. J. L. M. Bernink et al., “The TIMI risk score for unstable angina/non-ST elevation MI: a method for prognostication and therapeutic decision making," Journal of the American Medical Association, vol. 284, no. 7, pp. 835-842, 2000.

[14] S. D. Wiviott, E. M. Antman, C. M. Gibson et al., "Evaluation of prasugrel compared with clopidogrel in patients with acute coronary syndromes: design and rationale for the TRial to assess Improvement in Therapeutic Outcomes by optimizing platelet InhibitioN with prasugrel Thrombolysis In Myocardial Infarction 38 (TRITON-TIMI 38)," American Heart Journal, vol. 152, no. 4, pp. 627-635, 2006.

[15] Y. B. Song, J. Y. Hahn, H. C. Gwon et al., "A high loading dose of clopidogrel reduces myocardial infarct size in patients undergoing primary percutaneous coronary intervention: a magnetic resonance imaging study," American Heart Journal, vol. 163, no. 3, pp. 500-507, 2012.

[16] G. Patti, G. Bárczi, D. Orlic et al., "Outcome comparison of 600and 300-mg loading doses of clopidogrel in patients undergoing primary percutaneous coronary intervention for ST-segment elevation myocardial infarction: results from the ARMYDA-6 MI, (Antiplatelet therapy for Reduction of MYocardial Damage during Angioplasty-Myocardial Infarction) randomized study," Journal of the American College of Cardiology, vol. 58, no. 15, pp. 1592-1599, 2011.

[17] F. Mangiacapra, O. Muller, A. Ntalianis et al., "Comparison of 600 versus $300-\mathrm{mg}$ clopidogrel loading dose in patients with Stsegment elevation myocardial infarction undergoing primary coronary angioplasty," American Journal of Cardiology, vol. 106, no. 9, pp. 1208-1211, 2010.

[18] J. M. Siller-Matula, K. Huber, G. Christ et al., "Impact of clopidogrel loading dose on clinical outcome in patients undergoing 
percutaneous coronary intervention: a systematic review and meta-analysis," Heart, vol. 97, no. 2, pp. 98-105, 2011.

[19] G. Montalescot, L. Bolognese, D. Dudek et al., "A Comparison of prasugrel at the time of percutaneous Coronary intervention or as pretreatment at the time of diagnosis in patients with non-STsegment elevation myocardial infarction: design and rationale for the ACCOAST study," American Heart Journal, vol. 161, no. 4, pp. 650.el-656.el, 2011.

[20] L. Wallentin, R. C. Becker, A. Budaj et al., "Ticagrelor versus clopidogrel in patients with acute coronary syndromes," The New England Journal of Medicine, vol. 361, no. 11, pp. 1045-1057, 2009.

[21] G. Biondi-Zoccai, M. Lotrionte, P. Agostoni et al., "Adjusted indirect comparison meta-analysis of prasugrel versus ticagrelor for patients with acute coronary syndromes," International Journal of Cardiology, vol. 150, no. 3, pp. 325-331, 2011.

[22] E. M. Antman, D. T. Anbe, P. W. Armstrong et al., "ACC/AHA guidelines for the management of patients with ST-elevation myocardial infarction-executive summary: a report of the American College of Cardiology/American Heart Association Task Force on Practice Guidelines (writing committee to revise the 1999 guidelines for the management of patients with acute myocardial infarction)," Circulation, vol. 110, no. 5, pp. 588-636, 2004.

[23] E. M. Antman, M. Hand, P. W. Armstrong et al., "2007 focused update of the ACC/AHA, 2004 guidelines for the management of patients with ST-elevation myocardial infarction: a report of the American College of Cardiology/American Heart Association Task Force on Practice Guidelines," Journal of the American College of Cardiology, vol. 51, no. 2, pp. 210-247, 2008.

[24] F. G. Kushner, M. Hand, S. C. Smith et al., "2009 focused updates: ACC/AHA guidelines for the management of patients with ST-elevation myocardial infarction (Updating the 2004 Guideline and 2007 Focused Update) and ACC/AHA/SCAI Guidelines on Percutaneous Coronary Intervention (Updating the 2005 Guideline and 2007 Focused Update)," Journal of the American College of Cardiology, vol. 54, no. 23, pp. 2205-2241, 2009.

[25] J. L. Anderson, C. D. Adams, E. M. Antman et al., "ACC/AHA 2007 guidelines for the management of patients with unstable angina/non-ST-elevation myocardial infarction. A report of the American College of Cardiology/American Heart Association Task Force on Practice Guidelines (writing committee to revise the 2002 guidelines for the management of patients with unstable angina/non-ST-elevation myocardial infarction)," Journal of the American College of Cardiology, vol. 50, no. 7, pp. el-e157, 2007.

[26] R. S. Wright, J. L. Anderson, C. D. Adams et al., "2011 ACCF/AHA focused update of the Guidelines for the Management of Patients with Unstable Angina/Non-ST-Elevation Myocardial Infarction (updating the 2007 guideline): a report of the American College of Cardiology Foundation/American Heart Association Task Force on Practice Guidelines developed in collaboration with the American College of Emergency Physicians, Society for Cardiovascular," Journal of the American College of Cardiology, vol. 57, no. 19, pp. 1920-1959, 2011.

[27] H. Jneid, J. L. Anderson, R. S. Wright et al., "2012 ACCF/AHA focused update of the guideline for the management of patients with unstable angina/non-ST-elevation myocardial infarction (Updating the 2007 Guideline and Replacing the 2011 Focused Update)," Journal of the American College of Cardiology, vol. 60, no. 7, pp. 645-681, 2012.
[28] C. W. Hamm, J. P. Bassand, S. Agewall et al., "ESC Guidelines for the management of acute coronary syndromes in patients presenting without persistent ST-segment elevation: the task Force for the management of acute coronary syndromes (ACS) in patients presenting without persistent ST-segment elevation of the European Society of Cardiology (ESC)," European Heart Journal, vol. 32, no. 23, pp. 2999-3054, 2011.

[29] P. G. Steg, S. K. James, D. Atar et al., "ESC Guidelines for the management of acute myocardial infarction in patients presenting with ST-segment elevation The Task Force on the management of ST-segment elevation acute myocardial infarction of the European Society of Cardiology (ESC)," European Heart Journal, vol. 33, no. 20, pp. 2569-2619, 2012. 


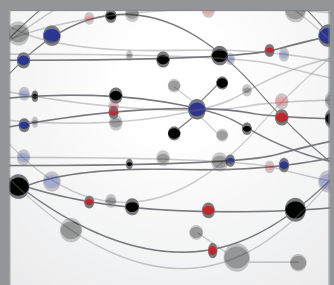

The Scientific World Journal
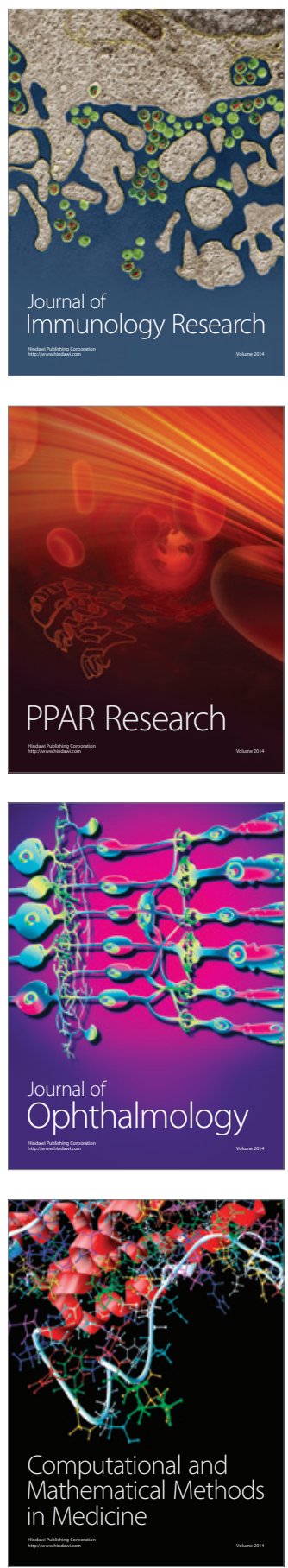

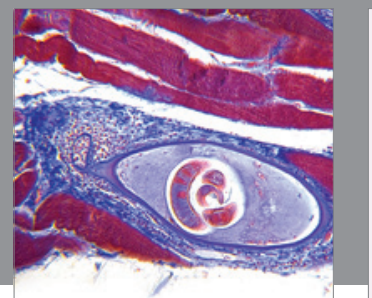

Gastroenterology

Research and Practice
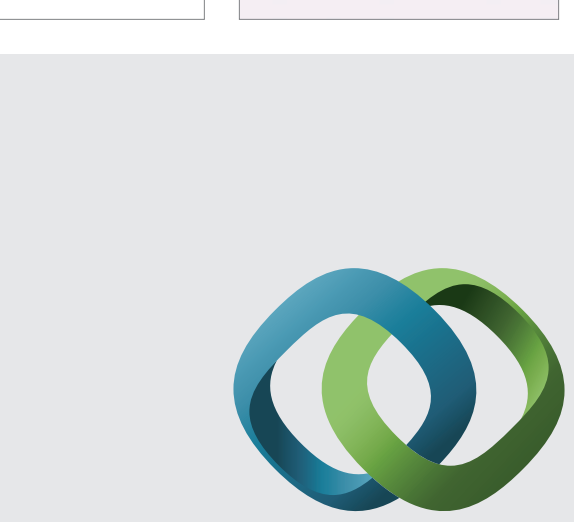

\section{Hindawi}

Submit your manuscripts at

http://www.hindawi.com
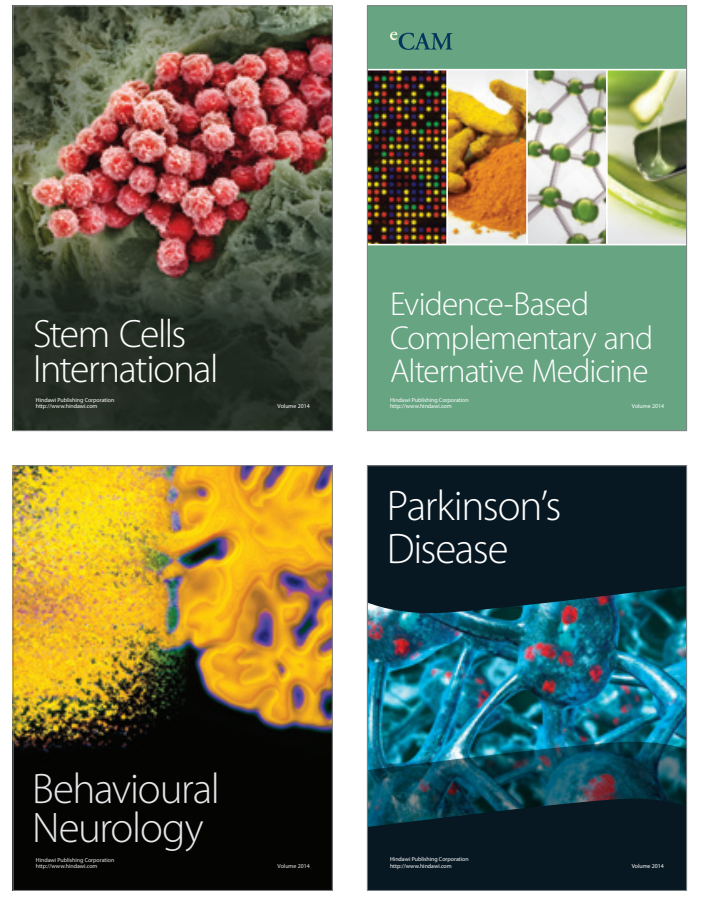
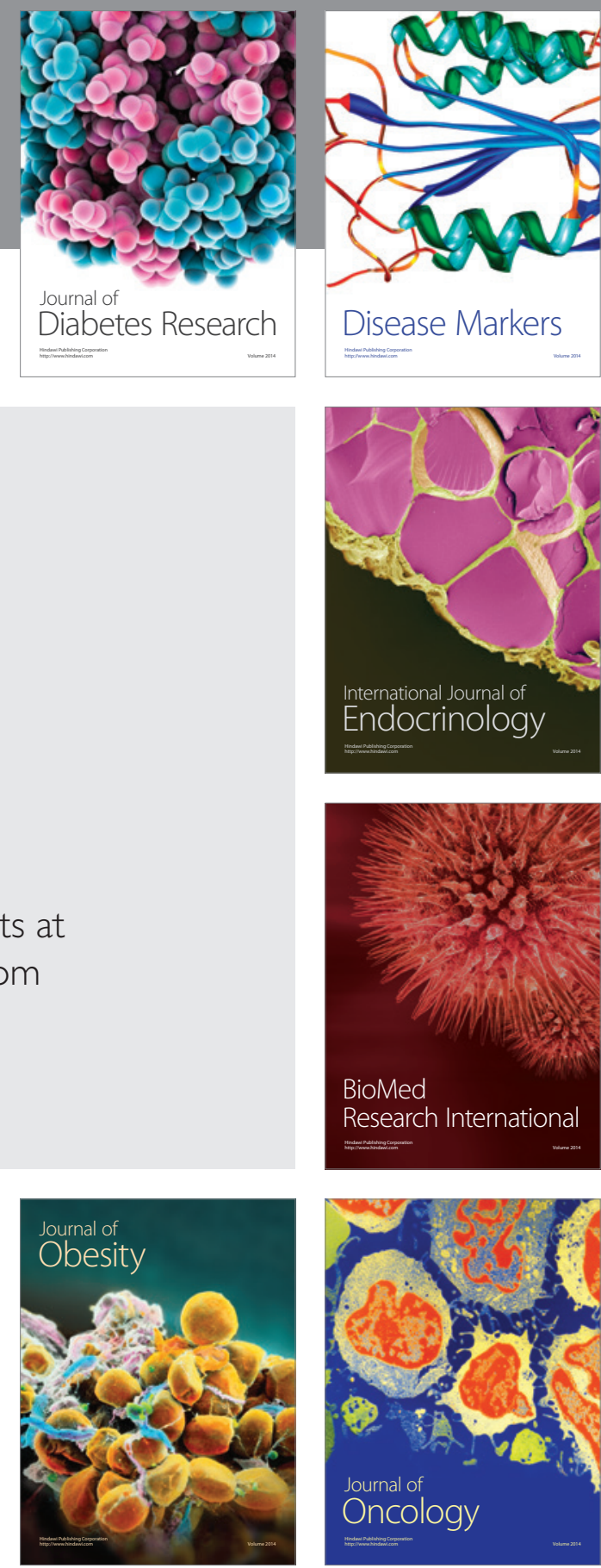

Disease Markers
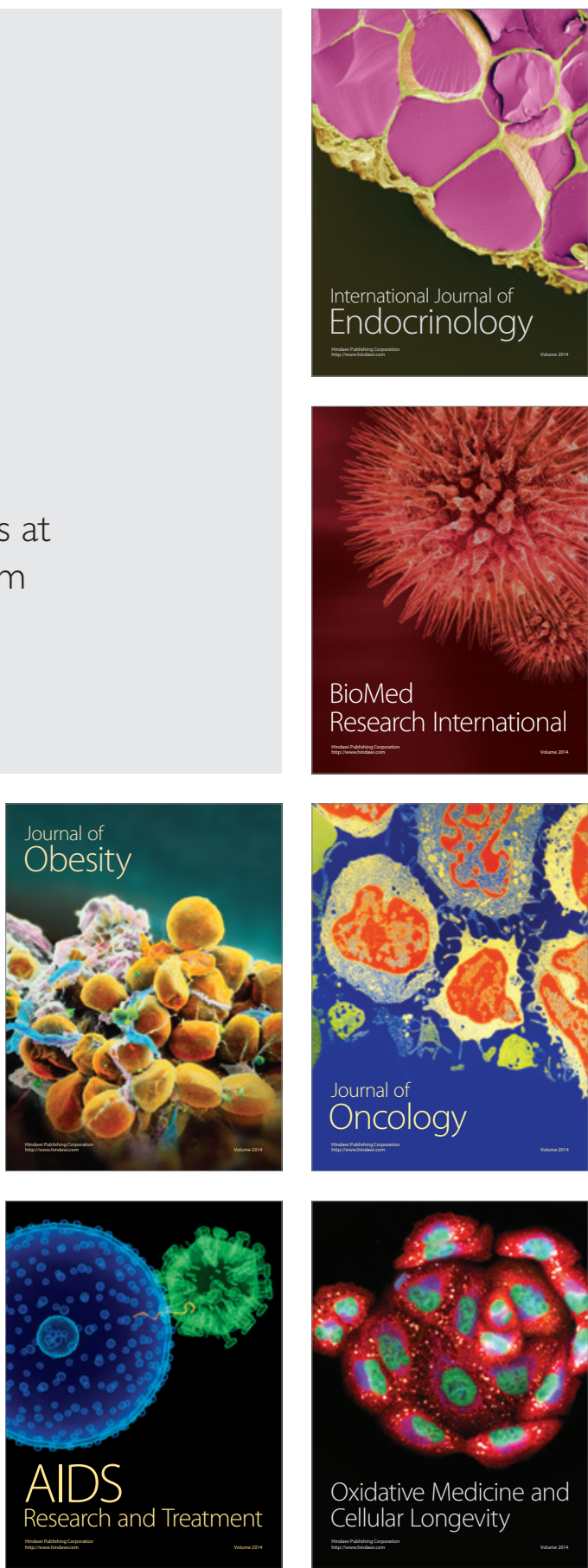